\title{
Reverse Engineering, Some Board Games as a Key to the Development of Artificial Intelligence and Construction of Economic Models
}

\author{
Ewa Drabik \\ Warsaw University of Technology, Warsaw, Poland
}

\begin{abstract}
Reverse engineering, also called back engineering, is the process of extracting knowledge or designing information from anything: mechanical device electronic components, computer programs, or biological, chemical matters, and - subsequently - analyzing its components. Reverse engineering can be applied for the sake of creating artificial intelligence, e.g. when the used methods are based on very old games, such as Go and chess. Many generations of computers are able to play at the same level as human grandmasters. A computers' arrival at that level is due to imitation of human Go or chess play. A particular emphasis shall be placed upon the Go game, known for 5,500 years. Invented in China, it may be classified as the oldest board game, having its ardent enthusiasts until present times. Old physical issues can be easily projected upon the modeling of new economic phenomena and quantum games. The aim of this paper is to discuss the applications of reverse engineering, traditional social games and other domains, such as quantum physics, to the analysis of utterly new social as well as economic phenomena.
\end{abstract}

Keywords: reverse engineering, category theory, quantum games, social games, modelling of economic phenomena

\section{Introduction}

Reverse engineering is a method of researching a product, device, program, human, artificial intelligence etc. in order to determine exactly how it functions, as well as how and with what resources it was created. The method is based on retracing a thought process backwards, in order to find the fastest way forward, usually to gather the information necessary to design a substitute, often superior to its original. Reverse engineering is applied when the attempts to build a device or solution aren't successful despite the work and resources consumed. It often also includes efforts to figure out an existing "device" that functions properly but it is unclear how it's constructed, for example due to lacking documentation. Another application of the method is to compare and ensure compatibility between products (Yurichev, 2016).

Another term that can be linked to reverse engineering is one rooted in sociology-the so-called "latecomer's advantage". An example of this phenomenon could be a country that starts developing a market economy later than others and so does not have to invent new technologies and other structures involved in democracy, such as effective governance models.

Ewa Drabik, professor, Faculty of Management, Warsaw University of Technology, Warsaw, Poland.

Correspondence concerning this article should be addressed to Ewa Drabik, Faculty of Management, Warsaw University of Technology, Narbutta 85, Warsaw 02-524, Poland. 
Reverse engineering is also used by the military in order to replicate the technologies prototyped by other countries, often supporting intelligence activities.

Reverse engineering can also be applied to financial markets in order to recreate the known mechanisms of the selling and purchasing of securities. Although the financial market is divided into many segments depending on the type of assets being circulated, the rules established by the "old" widely used technical analysis from one of the most effective models for profitable, often huge-scale transactions. It is worth mentioning that in this particular case the time-honored findings of social engineering also come into playing, as the changes in the investors' awareness and attitudes should be taken into account. Due to the uncontrollable actions of entities on any market, financial or otherwise, an oversell of financial instruments may occur causing a huge (and often unsuited for the current market situation) flow of capital, leading to the loss of market balance.

Reverse engineering can also be applied in game theory. Prisoner's dilemma, battle of the sexes, the game of chicken, or winner-takes-all games such as Blotto can be used to describe real market situations (Drabik, 2016). Many of those were devised shortly after World War II (e.g. the prisoner's dilemma was created in 1950 at RAND Corporation as a means of solving problems related to the Cold War). One of the most "effective" known economic balances is the Nash equilibrium, which proposes picking complementary strategies that together lead to the best solutions, even though accomplishing it doesn't necessarily provide the best possible outcome for the individual. It was designed over 70 years ago and no equally effective solution has been found since.

Social games, on which game theory is based, are even older. Chess, poker, baccarat, one-armed bandit, and positional games such as hex were fundamental to artificial intelligence research, the search for effective market strategies etc. Effectiveness in particular is important from an economic standpoint. Contrary to popular opinion, it is effectiveness, not money, that constitutes the central concept in economy. Evidence of this can be found for example in the Roth algorithms associated with "matching markets" . Chess has also served as a model for the creators of artificial intelligence ${ }^{2}$ and has contributed to the development of computers with ever increasing computing power.

Beginnings are often hard, and such was the case with creating chess based programs. The first automatic chess machines were built in the 18th century (e.g. a machine called the Turk). They were, however, a mere hoax designed to "deceive" the spectators. It was only in 1949 that Alan Turing developed the assumptions necessary for the development of the chess engine. In 1950, Claude Sharon built the Caissac machine, which among other things made it possible to play out chess endgames. Until the mid-1960s, chess programs were on the level of beginning players. In the 1970s, the Association for Computing Machines began systematically

\footnotetext{
${ }^{1}$ Matching markets - a so-called deferred acceptance algorithm created by Alvin Roth and Lloyd Shapley (Nobel Prize in Economic Sciences in 2012), based on a model in which two parties active on the market have very strong preferences as to who specifically (from the opposite side) they interact with. The decisions they make are based on the deletion from the preference list of those preferences that are not guaranteed to be fulfilled. A good example of a stable deferred acceptance algorithm is the "marriage market". It works in the following way: in the first round, each of the gentlemen participating in the "game" proposes to a lady who is on his list of accepted partners. Each of the ladies accepts the proposal of the gentleman whom she values most. Each rejected gentleman crosses out of the preference list the lady who rejected him, and in the next round the mechanism is repeated. With lists becoming limited, a gentleman who has crossed out all accepted partners no longer proposes. The mechanism ends when in the next round no one is rejected. The mechanism leads to a certain allocation in a finite number of steps and can be considered stable.

2 Artificial intelligence is a science concerned with developing and researching intelligent behavior algorithms.
} 
organizing chess program tournaments. The work of programmers dealing with the problem of chess automation included decision-making algorithms based on the movements of chess grandmasters, association memory, and image recognition. Only the increase in computing power brought the expected success. In the 1980s, Bell built a specialized chess computer that considered four moves forward. The next few years brought the improvement of programs capable of processing 750 thousand positions per second (deep thought created by IBM). In this case, parallel processing technology was used. In 1993, a computer "won" a match with the young chess master Judit Polgar. Another breakthrough turned out to be the multi-processor computer called Deep Blue, which was able to use specialized chess processes to generate a position, evaluate its value, and analyze further moves. In February 1996, in Philadelphia, the computer lost in its first duel with Gari Kasparov. However, in May 1997, in New York, it managed to beat him. The fact is that its advantage was in enormous computing power and the ability to quickly access an extensive database. For the analysis of chess positions it used a procedure called selective follow-up (alternative moves were investigated in a loop; if the position was judged to have a lower value the operation was interrupted and the program selected the move that it rated as having the highest value). Some computer technologies were based on Deep Blue's experience, i.e. DRMS (Distributed Resource Management System) — dynamic management of parallel environments and UTE (United Trace Environment) - universal rules for software running in parallel mode. In turn, parallel processing technologies are used in molecular dynamics, seismic analysis, meteorology, business intelligence which includes data mining, decision support, and knowledge management. In 2011, IBM also developed an intelligent computer capable of understanding human speech, called Watson (after IBM founder J. Thomas Watson). The machine can gather and search through a huge amount of information. It has won, among others, a duel with two champions of the American "Leopards" tournament. This computer assimilates a wealth of information from professional medical journals and quickly analyses thousands of specific cases. It presents the best options, based on which a physician can give a diagnosis. Now, a new generation of computers is being developed-quantum computers, i.e. ones that utilize a certain class of quantum phenomena. Those machines will be capable of autonomous decision making.

Ever since computer programs have "learned" to play chess effectively, technologies based on the algorithms they use allow countries that possess them (e.g. the USA) to impose their interests around the world. It is worth mentioning, however, that there is a place in the world where chess is sometimes played but there is much more passion for an even older game, Go (China), which is presented in chapter 2. Models of economic phenomena based on both classical and quantum physics are not new; physics problems that they use, which have been created decades ago for modeling a whole range of social and biological processes, can be considered as one of the elements of reverse engineering. Economic issues, modeled among others with the use of quantum mechanics, are discussed in chapter 3. Analogies between an English auction and quantum mechanics are presented in chapter 4 . Category theory, created over 70 years ago, is concerned with combining various disciplines, including the oldest ones; this is discussed in chapter 5.

\section{Research Design}

\section{Research Subject}

The paper aims at discussing about applications of traditional social games such a chess and Go and other domains such as quantum physics, to the analysis of utterly artificial intelligence, new social as well as economic phenomena. 


\section{Theory and Hypotheses}

Reverse engineering can be applied for the sake of creating artificial intelligence, e.g. when the used methods are based on very old games, such as Go and chess. Many generations of computers are able to play at the same level as human grandmasters. A computers' arrival at that level is due to imitation of human Go or chess play. Old physical issues can be easily projected upon the modeling of new economic phenomena and quantum games.

A growing number of economic phenomena are nowadays described with method, known in quantum mechanics (1935). Category theory has had a significant influence on many theories and introduces a systematic way of thinking about the general relationships between various objects, whether physical or social.

\section{Go Game as a Base of Artificial Intelligence}

An example of a board game much more unpredictable and therefore more difficult than chess is the game of Go. Go is one of the oldest games in the history of mankind. It was created in China in the years 2357-2255 BC. In the following centuries, it became the national game of the Japanese. In Europe Go became widely known in the 1890s, first in Germany, then other countries. It reached the USA in the 1930s. The elements necessary for the game are: a square board with a grid of 19 intersecting lines, and 360 pieces (called "stones"): 180 black and 180 white (see Figure 1). The rules of the game are as follows.

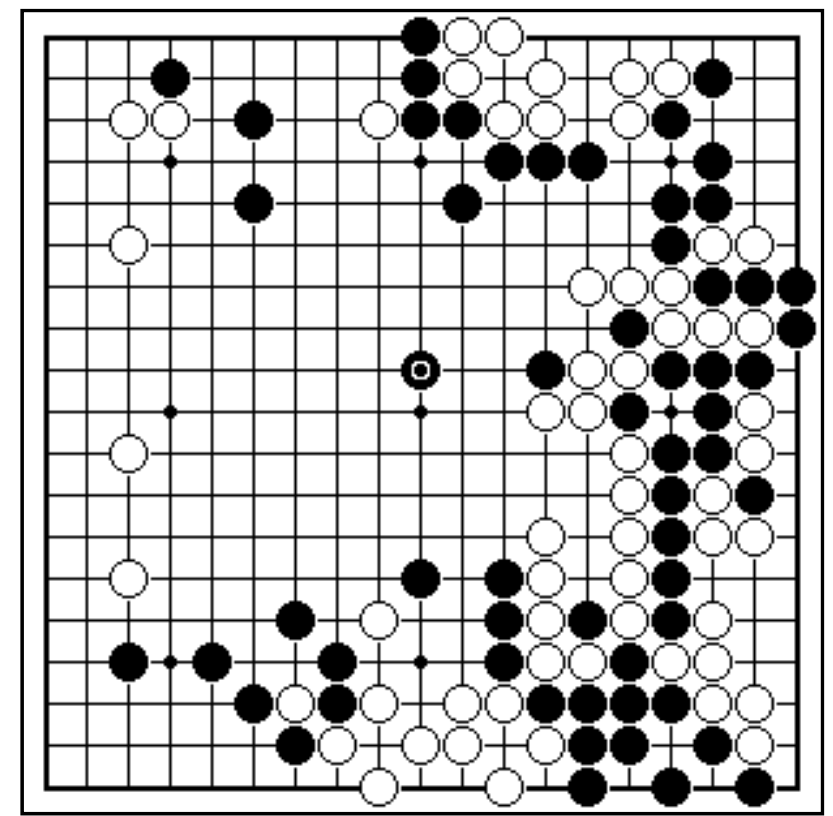

Figure 1. The Go board with pieces. Source: based on Pijanowski (1972).

Black moves first.

- The players take turns placing black and white stones on line intersections.

- The goal of the game is to use your stones to surround a larger territory on an initially empty board than your opponent - to gain the maximum amount of space.

- Stones, once placed on the board, may not be moved; they may however be captured by the opponent.

- Each stone placed on the board can have "liberties"-unoccupied neighbouring intersections linked to the stone with a straight line. Stones of the same color placed next to each other and connected with straight lines 
form a chain, which has shared liberties - the stones can either be captured all at once or not at all. The opponent can separate stones or chains with their own stones, cutting them off. If a stone or chain is captured, it is removed from the board.

- In the course of the game, when both players are successively placing new pawns on the board, linking them into chains, creating camps, and capturing the opponent's pieces, the board is gradually divided into white or black territories and border regions. The game is over if further moves (placing more stones) can no longer change the situation on the board.

As a result, a maximum amount of space on the board must be conquered (points are awarded for claiming space by forming chains of the player's own stones as well as surrounding and capturing the opponent's stones).

Looking at the course of the game, one can conclude that the strategy seems to make no sense, as opposed to chess which has known opening moves, defense strategies, etc. At first glance, no rational tactic is apparent, only to reveal a very complex and deliberate figure in a single move.

Despite the simplicity of its rules, Go is a very difficult game, at least for computers. That's why, when playing Go over the Internet, the likelihood that the opponent will use the help of a computer is minimal. There are also significant differences between players when it comes to strength and skill. Nevertheless, recent years have brought spectacular success in this field as well. Go has contributed to the development of computer programs, and thus artificial intelligence. In 2016, a computer called Deep Mind using the Alpha Go program defeated an 18-time Go world champion, Lee Sedola from Korea (score 4:1). Deep Mind, after playing many games (in other words, after numerous optimizations) made a connection between bouncing a stone and collecting points. The computer has "deduced" that it should "punch" a hole through the wall of the opponent's pieces, and then place a stone inside of the area surrounded by the opponent's stones. That stone, by bouncing off the edge of the board, will continue to eliminate the bricks in the wall. In this case, the computer did not defeat its opponent with computational power (it would be difficult even for machines with high computational power, since the number of possible moves in Go is higher than the number of atoms in the universe), but in a unique move which went against all known strategies developed by master players through thousands of years. The authors of the program used neural networks to enable self-learning (deep learning). Each game played was recorded in Deep Mind's memory and treated as data, based on which it changed its behavior in order to get as many points as possible.

In terms of game theory, Go is a positional combinatorial game with zero-sum information. Decisions (moves) create discrete outcome vectors. Each strategy is a function dependant on the current positioning of the stones. A position of a stone on the board can be represented in a relevant point in the game tree. Unlike the tactical game that is chess, Go is a strategy game. Chinese deliberately utilize strategies derived from Go for effective state management (often against their own citizens). These strategies, in contrast to ones used in other post-communist countries, have been showing satisfactory results so far. Therefore they are worth examining in detail, in order to be applied in the social sciences. Additionally, much like in the case of chess, strategies derived from Go are the foundation of not only management, but also of artificial intelligence. Artificial intelligence is increasingly used in the power industry, in the creation of systems monitoring specific processes, in planning, and in decision making. It is also used for image processing (e.g. in cameras), financial decisions support, and many other areas of everyday life. 


\section{Economic Measurement Precision and Connections to Quantum Physics}

Since the 1960s, economic phenomena have been modeled using gravity models, as well as static physics models (so-called minority games-1990s). Models based on the laws of classical physics have some disadvantages, though. In this case a crucial point is the approach to concepts well rooted in economics, such as risk and uncertainty. There are many ways to define risk; one of them is that certain economic activities will end in failure. In turn, uncertainty is associated with insecurity, incomplete and unequal access to information. From the point of view of contemporary economic theory, risk can be measured, for example by means of dispersion measurement; meanwhile, uncertainty can not be measured. That is why, at the beginning of the 21 st century, theorists turned to quantum physics and developed, among other things, quantum game theory. An important law of quantum mechanics is the Heisenberg principle of uncertainty (non-determinism), which in simplistic terms states that two different physical properties of a studied object can not be simultaneously determined with equal accuracy. In physics, measurement is understood as a process in which an instrument interacts with the investigated object, as a results of which information is obtained about the object's properties. In many cases measurement itself has a significant impact on the observed process and serves to assess a whole range of phenomena. The act of investigating may distort the result. Heisenberg's claim essentially consists in the fact that theory does not need to introduce into the representation of reality any elements that are not observable. According to classical physics, any physical phenomena can be measured with any precision. It is impossible, however, to experimentally confirm or deny the accuracy of these calculations with $100 \%$ certainty. Quantum theory, in turn, does not say much about the specific location of an object in space. Instead, it determines the probability of finding it in a certain point in space. It determines the precision of the measurement, similarly to the whole series of what's known as quantitative methods, used in social and economic sciences (Feynman, Leigton, \& Sands, 1972).

An important principle in quantum theory is the principle of W. Paulli stating that two objects can not occupy the same place at the same time. This principle in economic sciences can be regarded as a law. Meanwhile the uncertainty principle, as a universal law, turns out to be applicable in many areas as mere observation of economic processes is not enough to perform unambiguous measurements and then quantify them. Even more difficult is making economic decisions in conditions of uncertainty. Various statistical methods are used for this purpose, as well as mathematical models based e.g. on neural networks, genetic algorithms, or chaos theory. These methods generate approximate results, and the measurement itself is enough to distort the result. As was previously mentioned, according to the Heisenberg principle it is not necessary to introduce elements that are not observable. For example, the inference on the basis of statistics is influenced in turn by: the means of collecting data, the method of measurement (i.e. planning of the cognitive experiment), sample selection, elimination of distortions and uncontrollable factors, and many others. This is illustrated by the following example.

\section{Example}

The secretary problem (Moore, 1983). A group of $N$ candidates for vacant secretary position differ in qualifications: from 1 - best candidate to $N$-worst candidate, in random order, so that the best one has equal chances of applying as first, second, $k(k=3,4, \ldots, N-1)$, or last. After interviewing each next candidate, the interviewer can judge the candidate's qualifications without error, but they must immediately make a decision: either reject or accept the applicant. The decision is final and there is no going back to the previous candidates. The problem is to find the procedure that gives the best chance of getting the best candidate. 
This problem belongs to the group known as optimal stopping problems. There is a whole range of methods to solve it. One can, for example, apply the rule of reverse induction. In this case the reasoning is performed backwards. There is a $1 / N$ probability that the candidate will apply last. Interviews can be stopped after $N-1$ steps or after $N$ steps. If candidate $N-1$ is not the best out of the $N-1$, then stopping at this point does not give any chance of choosing the best candidate. In this case, one should continue the interviews relying on the $1 / N$ probability that the last candidate will be the best one. However, if candidate $N-1$ is the best among the respondents interviewed so far, the situation changes.

Reverse induction allows stopping the interviews after candidates $N-2, N-1$, or $N$ and compares the chance of success after $\mathrm{N}-2$ conversations with chances of success after continued interviews. The principle imposed by reverse induction states that first a fixed number of candidates must be omitted and then the first candidate to be best out of all interviewed thus far should be employed. For a large $N$, the principle dictates to omit about $37 \%$ of the candidates. For example, for $N=4$, the rule omits the first candidate and then selects the next one that is better than all the preceding candidates. If the candidate was the best, the last one is selected. All possible arrangements in which candidates can apply for interviews are in Table 1.

Table 1

Four Persons: Secretary Problem

\begin{tabular}{llll}
\hline 1234 & $* 2134$ & $* 3124$ & $* 4123$ \\
1243 & $* 2143$ & $* 3142$ & $* 4132$ \\
1324 & $* 2314$ & 3214 & 4213 \\
1342 & $* 2341$ & 3241 & 4231 \\
1432 & $* 2413$ & $* 3412$ & 4312 \\
\hline
\end{tabular}

Note. * - the rule selects the optimal candidate. Source: Moore (1983).

Let candidate 1 be the best, 4 the worst. Digits in bold signify the candidate who will be selected in each arrangement after following the optimal rule. An asterisk signifies the arrangements for which the rule selects the optimal candidate ( 11 out of 24 cases). Therefore the optimal rule gives a success rate of $11 / 24 \cong 0.46$. A random drawing gives success probability of $1 / 4$.

It can therefore be noted that in the case of the secretary problem, the "measurement" can not be accurate, and it is not always possible to precisely locate the candidate irrespective of the method used. This is also the case in physics, which confirms that the Heisenberg method is universal. The essence of the problem lies in the fact that accepting or rejecting subsequent applications will not always result in the best selection. Similarly in physics it is impossible to accurately measure in particular several physical properties simultaneously, which is the essence of the Heisenberg principle. The use of "better" measuring instruments has no significant impact.

The previously mentioned neural network also operates on the basis of approximation. Although it has the capability to learn and possibly to generalize the acquired knowledge, the approximation in this case is even greater, and additionally contradictory signals can generate contradictory decisions. Artificial neural networks are very popular in modeling control systems of artificial organisms. In experiments, it is expected that with the use of neural networks the "answer" will be repeated and updated many times as needed. The problem, however, is the learning ability of a network which is still under the control of the optimization algorithm.

Genetic algorithms, in turn, are based on the imitation of evolution. They test certain rules and select from among them a set of rules that optimize a particular function. The use of prognostic methods based on genetic 
algorithms requires the accumulation of large quantities of initial data, which is not always feasible, creating a high risk of errors at the very outset. Chaos theory, also used in economics, is based on the premises of evolution of deterministic systems which may behave in a chaotic manner in certain situations. These phenomena are generally described by nonlinear systems. If nonlinear systems are involved, then in physics they can be described by the Schrödinger equation, which scientists consider to be an almost perfect illustration of quantum physics phenomena. This equation may prove to be a good prognostic tool in social sciences. It is worth mentioning that Schrödinger modeled it based on the existing laws of the macro-, not micro-world. He took advantage of the properties of thermal waves. This equation of his has been applied multiple times, e.g. in the research for the famous Manhattan project (Ulam, 1996).

Those who know and understand this equation in depth are capable of making precise predictions. In the worst case, this equation lets one conclude that if the probability of finding an object at a certain point in space increases, and decreases for another point, so that the sum of probabilities does not change, then there must be some interaction between these points. This observation seems crucial also when it comes to economic theory.

Approximate results that "overlap" cause the multiplication product of the errors associated with a prognosis or a decision taken is not smaller than a fixed value characterizing a particular phenomenon. This constant can be treated as an economic constant analogous to the Planck constant.

Classical theory of both physics and economics, referring to a particular phenomenon, assumes that if it "exists", it is "as we see it". The truth, however, is that this "existence" takes place on many levels. It turns out that a good explanation of this state of affairs is the Schrödinger's cat paradox (1935). It is known from quantum mechanics that, according to state superposition, particle parameters assume all possible values allowed by physics until the moment the measurement is carried out. The measurement (observation) leads to the transformation of superposition into real state. According to latest theories, superpositions do not break into one state constituting reality. Scientists claim that there is a division of these "realities" at the moment of each quantum event and that jumps into "paralled realities" may occur. In general, this is how we can present the loss of the ability to anticipate events when moving from one state to another. Schrödinger gave the example of a cat trapped in a box. The cat could either be killed by electromagnetic or radioactive substance discharges or survive. Cat may either die or remain alive. The chance that it will survive amounts to 50 percent. According to Schrödinger, quantum mechanics implies that the cat remains both alive and dead (to the universe outside the box) until the box is opened, which may be described as follows:

$$
\left.\left.\left|\Psi_{\text {cat's }}\right\rangle=\alpha \mid \text { alive }\right\rangle+\beta \mid \text { dead }\right\rangle
$$

where $|\alpha|^{2}+|\beta|^{2}=1,\left|\Psi_{\text {cat's }}\right\rangle$ is the cat's state.

Having opened the box, i.e. having measured the system, an external observer will find the cat either alive or dead. At that moment the "cat's state" is reduced to one of the possibilities: $\mid$ alive $\rangle$ or $\mid$ dead $\rangle$. The experiment may be made even more complex by placing an internal observer in one of the box corners who will watch the situation from the inside. The "cat's state" $\left|\Psi_{\text {cat's }\rangle}\right\rangle$ is observed and then reduced to one of its components: $\mid$ alive $\rangle$ or $\mid$ dead $\rangle$, which may be visualized as the state of: |happiness $\rangle$ or $\mid$ sadness $\rangle$, respectively. On the other hand, an external observer describes the entire system containing an internal observer in the form of a linear superposition:

$$
\left.\left.\left.\left.\left|\Psi_{\text {cat's }}\right\rangle=\alpha_{1} \mid \text { alive }\right\rangle \otimes \mid \text { happiness }\right\rangle+\beta_{1} \mid \text { dead }\right\rangle \otimes \mid \text { sadness }\right\rangle
$$

where $|\alpha|^{2}+|\beta|^{2}=1$. 
The external observed will reduce the superposition concerned only after opening the sealed box. The idea is that whereas an external observer perceives the "cat's state" as a superposition of two states (dead-alive), the internal observer may experience either happiness due to the cat's survival or sadness due to its death.

These laws of quantum physics have been applied to quantum game theory. The choice of strategy in a classic game very often corresponds to a zero/one choice, which means that the player either chooses or rejects a particular strategy, so it operates on bits. The laws of quantum physics allow one object to be in two states at the same time. Schrödinger's cat can be both alive and dead, and a q-bit, unlike an ordinary bit, can have both the values of zero and one. It is only by interacting with other objects that the q-bit "decides" to choose one of the possibilities. A similar description applies to the phenomenon of decoherence, which results in the measurement results being independent of each other.

Similar analogies may be proliferated, however, at least one more deserves special attention, i.e. Schrödinger's cat. Schrödinger's cat paradox can be applied, for example, in an auction model. Consider, for example, dynamic auctions: an English auction (the price of the auctioned item is increased sequentially, after the acceptance of the "current" price is quoted by the auctioneer by one of the buyers, until there is no buyer willing to give more) or Dutch auction (the price of the item is reduced until it is accepted by a buyer). Simple analogies may be proliferated with dynamical auction (Drabik, 2011a).

The state of the seller $(k=0)$ before the transaction completion (i.e. the measurement) may be described as follows:

$\left|\Psi_{0}\right\rangle=\alpha \mid$ transaction completion $\rangle+\beta \mid$ failure to complete the transaction $\rangle$ where $|\alpha|^{2}+|\beta|^{2}=1$.

The state of purchaser $k(k=1,2, \ldots, N)$ may be presented as follows:

$\left|\Psi_{k}\right\rangle=\alpha_{1} \mid$ satisfaction from the transaction completion $\rangle \otimes \mid$ transaction completion $\rangle+\beta_{1} \mid$ dissatisfaction from a failure to complete the transaction $\rangle \otimes \mid$ failure to complete the transaction $\rangle$ where $|\alpha|^{2}+|\beta|^{2}=1$.

The states specified in above formulas correspond to "diagnoses" of the external and internal observers in Schrödinger's cat thought experiment. When the auction is completed, the initial state $\left|\Psi_{k}\right\rangle_{\text {out }}$ is reduced to the state: $\mid$ transaction completion $\rangle$ or $\mid$ failure to complete the transaction $\rangle$, where purchaser $k(k=1, \ldots, N)$ experiences $\mid$ satisfaction $\rangle$ whenever the product is purchased on profitable conditions, or $\mid$ dissatisfaction $\rangle$ in the opposite case.

Similar situations are very commonplace on financial markets, where diversified access to information illustrates the current situation of the investor (see e.g. strong and weak market performance). The clusters of diverse strategies (for example, based on technical or fundamental analysis) that affect market mechanisms can be compared to waves, or rather a cumulation of waves. Thus, the wave-particle duality derived from quantum mechanics is also applicable in the social sciences.

Quantum game theory is particular in that it allows one to find a set of strategies better than those guaranteed by the Nash equilibrium in classical games. Uncertainty occurs both in classical and quantum games. In the case of the former, it is unknown how the opponent will behave. However, the rules for determining the Nash equilibrium are predetermined. In this case, the Nash equilibrium will be such a result of the game which guarantees the best (meaning beneficial to all) solution. The right balance should allow the players to recognize and respond appropriately to this uncertainty. To illustrate the point further, a choice of strategy in a classic 
game corresponds to a zero-one choice, which means that the player either decides to reject a strategy or use it. In most cases for mixed strategies there is a Nash equilibrium. The extended Nash equilibrium, i.e. quantum response equilibrium provides each player with a payout at least as high as in the classic game, and although it always exists it need not have a set of clean strategies in balance - even for zero-sum games (Landsburg, 2004). As previously mentioned, the laws of quantum physics allow for one object to be in two states at the same time. Most classic games can be modified to become quantum games. This applies to the prisoner's dilemma, battle of the sexes, and to such classics as rock-paper-scissors.

Quantum games are increasingly used in financial market theory, in the construction of quantum computers, in cryptography, in quantum algorithms. They are essentially based on a theory formulated years ago (Piotrowski \& Sładkowski, 2002). The same is true of the theory that aims to combine many scientific disciplines, namely category theory (Mayer, 1999).

\section{A Few Remarks on Category Theory and Its Practical Applications}

Category theory, created in the mid-twentieth century, deals with the relationships between different mathematical objects. It is also a theory of analogies between different sciences. From a formal point of view it is treated as an alternative to set theory. Both language and category theory methods have unified and simplified many concepts of logic, theoretical computer science, topology, and algebra.

Eilenberg and Mac Lane (1945) created the basis for category theory, based on the premises they had heard at the lectures of their teachers Stanislaw Ulam (in 1930 Eisenberg listened to his lectures in Lviv) and Emma Noether (Mac Lane's teacher). In this case, objects and their changes are considered as opposed to just the objects. It follows that the basic concepts are objects and their morphisms.

Objects can be simple, but they can also be sophisticated mathematical structures. The relationships between objects are considered; much can be learned about the structure of the objects while studying morphisms.

Category theory was further developed by Louis Carmen. He assumed that processes can occur not only between objects, but also between morphisms. Many important problems can be formulated in the form of questions about the classification of structures of a given type with isomorphism accuracy. Typically, attempts are made to classify structures relative to equivalence relations. Defining such equivalence relations involves assigning to the structure an invariant in the form of a number that is the same for two isomorphic structures (i.e. a number that is the same for the structures considered), e.g. by defining a certain kind of algebraic curve, a vector space dimension.

The idea has been systematized by defining a functor transporting isomorphic classes onto corresponding classes. Thus, the appropriate equivalence relation means that the imposition of such a functor on two objects results in isomorphic objects. Another way to treat a set of objects or class sets is to organize them by defining a certain kind of structure on such a set.

Category theory has had a significant influence on many theories, particularly in mathematics. It has introduced a general model, similar to that provided by set theory. In addition, it has introduced a policy never to define objects without simultaneously defining the processes that occur between those objects. It was also assumed that axiomatic set projections should be treated as a particular type of morphism.

An example of this can be ergodic theory, which was formulated as a discipline only after probability theory had been absorbed by measure theory. The invariant of this theory can be entropy. 
Different fields use different mathematical models to describe the same or similar processes. Category theory introduces a systematic way of thinking about the general relationships between various objects, whether physical or social. In this case, it is the relationships that are important, and by studying relevant "morphisms" we can learn much about the structure of the transformed objects.

\section{Conclusions}

It seems natural that we should use the knowledge of the past generations. Therefore reverse engineering appears to be the science of the future, not the past. The reverse engineering is analysed in order to deduce design features from products. Value engineering is a related activity also used by business, a taxonomy scheme, which referred the classifying of things or concepts.

It is also worthwhile to look to the achievements of the past, even ancient ones (board games).

The modeling of economic phenomena based on classical physics and quantum theory provides a more precise description than the classical probability calculus.

Old physical issues can be easily projected upon modeling of new economic phenomena and quantum games. This theory states that the natural phenomena may be described in approximation; each measured value is mistake-prone and falls within a specific range of probability. It assumes that microobjects may be located at several places simultaneously until they measured. This property has its own "economic" analogy, since the branch offices of a significant corporation may be placed in separate corners all over the world. Quantum game based on the theory of quantum mechanics, is an extension of a classical theory game. It has been developing since the beginning of 21 st century. Quantum games currently enjoy their increasing popularity. They are applied to, i.e. the theory of financial markets, the construction of quantum computers, quantum algorithms, cryptography, data transfer, theory of auctions, or examination of decoherence.

Attempts to merge many long-established disciplines had been made much earlier. The first one to prove effective was Kurt Gödel's attempt to combine various branches of mathematics. Already in 1931 he proved that all deductive systems, provided that they are not contradictory, are essentially incomplete. This incompleteness is important also from the point of view of other sciences, since it can not be removed by adding new axioms, and no single system can include the whole truth. Therefore it can be postulated that both reverse engineering and category theory have a great future ahead. The same reasoning can be applied to mathematical models of economic phenomena. Gödel has managed to determine one thing, namely an unambiguous correspondence between the formulas of the "language" of a given formal theory and natural numbers. At this point one might quote a parable drawn from antiquity. Plato (427 BCE-347 BCE) left an "encoded message" for posterity: "It is not Zeus, but numbers that govern the Universe". He had to use a cipher because his teacher, Socrates (470 BCE-399 BCE) had been sentenced to death for a far lesser offence against the Gods.

\section{References}

Drabik, E. (2011a). On certain analogies between the laws of quantum mechanics and rules of an English auction. Folia Oeconomica Stetinensia, 10(18), 219-237.

Drabik, E. (2011b). Classical and quantum physics in selected economic models. Foundation of Management, 13(1), 7-20.

Drabik, E. (2016). Several remarks on the role of certain positional and social games in creation of the selected statistical and economic applications. Foundation of Management, 8, 289-296.

Eilenberg, S., \& Mac Lane, S. (1945). General theory of natural equivalences. Transaction of the American Mathematical Society, 58, 231-294.

Feynman, R. P., Leigton, R. B., \& Sands, M. (1972). Quantum mechanics. Warsaw: WNT. 
Mayer, D. A. (1999). Quantum strategies. Phys. Rev. Lett., 82, 1052-1055.

Moore, D. S. (1983). Statistical analysis of empirical data. In L. A. Stern (Ed.), Mathematical today: Twelve informal essays (pp. 230-260). Warsaw: WNT (in Polish; original work published in 1979). New York, Heidelberg, Berlin; Springer-Verlag.

Landsburg, S. E. (2004). Quantum game theory. Notices of the AMS, 51(4), 394-399.

Pijanowski, L. (1972). Game guide. Warsaw: Iskry. (in Polish)

Piotrowski, E. W., \& Sładkowski, J. (2002). Quantum bargaining games. Physica A: Statistical Mechanics \& Its Application, 256, 514-532.

Ulam, S. M. (1996). The adventures of mathematician. Warsaw: Prószyński i S-ka. (in Polish)

Yurichev, D. (2016). Reverse engineering for beginners. Retrieved from: http://beginers.re/REYB-EN.pdf

https://www.nobelprize.org/nobel_prizes/lists/year/?year=2012

http://www.staff.science.uu.nl/ costel10/syllabi/catsmoeab.pdf

http/www.tac.mta.ca/tac 